\title{
Asymmetrical Flux Density Distribution in Stator Teeth of Surface Permanent Magnet Machines
}

\author{
Giacomo Sala, Daniele De Gaetano, Michele Degano, Chris Gerada
}

\begin{abstract}
This work is showing in detail the flux density behaviour in the stator teeth of a synchronous machine. A 3-phase Surface Permanent Magnet (SPM) motor is considered. These motors are widely employed in applications where high efficiency and power densities are required. This paper aims to analytically demonstrate the asymmetrical distribution of the stator teeth flux density. It is shown that this phenomena is depending on the number of slots per pole and per phase in the machine. Finally, a comparison with Finite Element results is given to validate the effectiveness of the proposed model.

Index Terms-Analytical models, Electric machines, Magnetic flux density, Permanent magnet machines, Rotating machines, Stators.
\end{abstract}

\section{INTRODUCTION}

FOR any machine design, the computation of the iron losses is a very important aspect for improving the machine efficiency and thermal management [1]. This is strictly related to the estimation of the flux density distribution and its behaviour with respect to different harmonic components. Several works in literature are proposing techniques to minimise the iron losses both analytically and via finite element optimisation. In [2], a method for reducing the harmonics due to the permanent magnets is proposed. Therefore, the flux density analysis is essential to estimate and minimise the iron losses at the design stage [3]-[5]. Other works [6], implement complex subdomain models to predict the flux density within the stator core always considering symmetrical behaviour [7]. In this paper, the flux density distribution in different stator teeth has been analysed in detail considering a distributed winding with a single layer and full pitch. Such winding configuration is often chosen in fault-tolerant electrical machines [8], [9]. First, an analytical solution has been implemented in section II, to identify eventual asymmetries in different parts of the stator core. This has been compared with Finite Elements results, presented in section III, for validation. Finally, a summary of the paper outcomes is offered in the conclusions.

\section{ANALYTICAL MODEL}

The model proposed in this paper is based on the assumption of radial flux density in the air-gap, no slotting effect and linear materials [10]. Under these hypotheses, the magnetic field in the air-gap can be evaluated for each angular position $(\vartheta)$ in terms of Fourier series as:

$$
H(\vartheta)=\sum_{\rho=1}^{\infty} \Re\left\{\left(\bar{H}_{S, \rho}+\bar{H}_{P M, \rho}\right) e^{-j \rho \vartheta}\right\},
$$

G. Sala, D. De Gaetano, M. Degano and C. Gerada are with the Power Electronics, Machines, and Control (PEMC) group, The University of Nottingham, Nottingham, UK.

M. Degano and C. Gerada are with The University of Nottingham, Ningbo, China.

This work was also partially funded by the University of Nottingham Propulsion Futures Beacon through Grant PF045.
TABLE I

MACHINES PARAMETERS

\begin{tabular}{cccccc}
\hline Slots & Phases & $\mathrm{p}$ & $\mathrm{q}$ & air-gap & magnet thickness \\
\hline 36 & 3 & 2 & 3 & $1[\mathrm{~mm}]$ & $4[\mathrm{~mm}]$ \\
12 & 3 & 2 & 1 & $1[\mathrm{~mm}]$ & $4[\mathrm{~mm}]$
\end{tabular}

where $\bar{H}_{\rho}\left(\bar{H}_{\rho}=\bar{H}_{S, \rho}+\bar{H}_{P M, \rho}\right)$ is the $\rho^{t h}$ Fourier series component of the spatial distribution of the magnetic field in the air-gap. The $\rho^{t h}$ harmonic contribution produced by the stator currents $\bar{H}_{S, \rho}$ can be defined as:

$$
\bar{H}_{S, \rho}=\frac{3 N q}{\pi \delta} \frac{K_{a \rho}}{\rho} \bar{i}_{\rho}^{S},
$$

where $N$ is the number of turns per phase, $q$ the slots per pole and per phase, $\delta$ the air-gap thickness and $K_{a \rho}$ is the winding factor for the $\rho^{t h}$ field harmonic. $\bar{i}_{\rho}^{S}$ is the $\rho^{t h}$ current space vector defined by the Clarke transformation of the currents for the three-phase winding $(\mathrm{U}-\mathrm{V}-\mathrm{W})$ considered:

$$
\bar{i}_{\rho}^{S}=\frac{2}{3}\left(i_{U}+i_{V} e^{j \rho \frac{2 \pi}{3}}+i_{W} e^{j \rho \frac{4 \pi}{3}}\right), \rho=0,1, \ldots \infty,
$$

where $j$ is the unity imaginary number $\left(j^{2}=-1\right)$.

Because the zero sequence current is null, due to the star connection of the three-phase winding, it is possible to write the following relationships among the space vectors:

$$
\begin{array}{ll}
\bar{i}_{\rho}^{S}=\bar{i}_{1}^{S} & \text { if } \rho=3 n+1, \\
\bar{i}_{\rho}^{S}=\bar{i}_{1}^{S *} & \text { if } \rho=3 n-1, \quad n=0,1,2, \ldots, \infty . \\
\bar{i}_{\rho}^{S}=0 & \text { if } \rho=3 n .
\end{array}
$$

Finally, the rotor magnets contribution, to the $\rho^{t h}$ harmonic of field in the air-gap, can be expressed with a good approximation as:

$$
\bar{H}_{P M, \rho}=\frac{4 B_{r} \delta_{P M}}{\mu_{P M} \pi \delta} \frac{\sin \left(\rho \Delta_{P M} / 2\right)}{\rho} e^{j \rho \vartheta_{r}},
$$

where $B_{r}$ is the remanence flux density, $\delta_{P M}$ the magnet thickness, $\mu_{P M}$ the magnet permeability, $\Delta_{P M}$ the magnet angular width, and $\theta_{r}$ the rotor position in electrical radians. Table I is summarising the main machine parameters. The airgap flux density, for the considered SPM machine, when the rotor is aligned with the magnetic axis of phase $\mathrm{U}\left(\theta_{r}=0\right)$, is shown in Fig. 1. The figure highlights the two components of the flux: the one produced by the magnets, and the flux generated by the stator currents, when the machine is fed with its rated current $\left(\bar{i}_{\rho}^{S}=j i_{q} e^{j \vartheta_{r}}\right.$, with $\left.i q=683 A_{p k}\right)$. The total flux density is obtained by the sum of two components. In order to evaluate the flux density in each stator tooth, the proposed model considers that all the flux, under the slot pitch, is crossing the air-gap and flowing through the same tooth. 


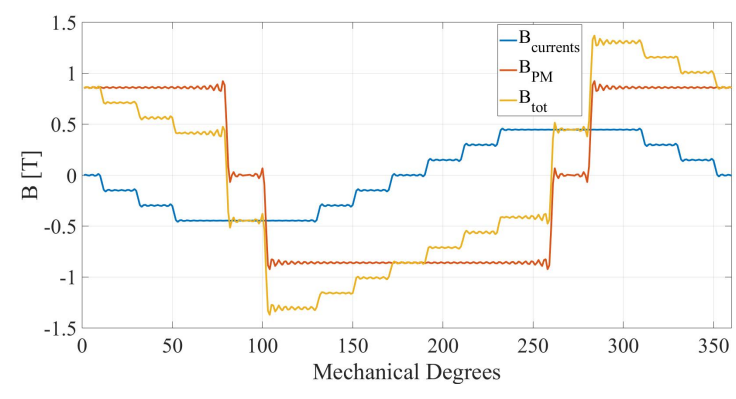

Fig. 1. Analytical flux density evaluation in the air-gap of a 3 slots per pole and per phase SPM machine. The initial rotor reference position is $\vartheta_{r}=0$ and the stator currents corresponding to the rated condition (100 spatial harmonics are considered)

Therefore, the analytical evaluation of the teeth flux is provided for each $T^{t h}$ tooth by:

$$
\Phi_{T}=\int_{\vartheta_{T}-\frac{\Delta_{s l o t}}{2}}^{\vartheta_{T}+\frac{\Delta_{s l o t}}{2}} \mu_{0} H(\vartheta) L R_{g} d \vartheta
$$

with $\vartheta_{T}=\frac{2 \pi p}{N_{\text {slots }}}(T-1)$ the angular position of the centre of the $T^{\text {th }}$ tooth ( $N_{\text {slots }}$ is the overall slot number of the stator), $\Delta_{\text {slot }}=\frac{2 \pi p}{N_{\text {slots }}}$ the angular pitch between two neighbouring slots (or teeth), $L$ the active length of the machine, and $R_{g}$ the middle radius of the air-gap.

Substituting (1), with (2) and (5), in (6) it is possible to define the $T^{\text {th }}$ tooth flux in terms of Fourier series as:

$$
\Phi_{T}=\sum_{\rho=1}^{\infty} \Re\left\{\bar{\Phi}_{T \rho}\right\}
$$

with:

$$
\bar{\Phi}_{T \rho}=2 \mu_{0} L R_{g} \frac{\sin \left(\rho \frac{\Delta_{\text {slot }}}{2}\right)}{\rho}\left(\bar{H}_{S, \rho}+\bar{H}_{P M, \rho}\right) e^{-j \rho \vartheta_{T}}
$$

Under the assumption of the model, the flux density related to one tooth can be evaluated as:

$$
\begin{aligned}
B_{T}= & \frac{\Phi_{T}}{w_{\text {tooth }}}=\frac{2 \mu_{0} L R_{g}}{w_{\text {tooth }}} \\
& \sum_{\rho=1}^{\infty} \frac{\sin \left(\rho \frac{\Delta_{\text {slot }}}{2}\right)}{\rho} \Re\left\{\left(\bar{H}_{S, \rho}+\bar{H}_{P M, \rho}\right) e^{-j \rho \vartheta_{T}}\right\},
\end{aligned}
$$

Eq. 9 can be rewritten in terms of Fourier series as:

$$
B_{T}=\sum_{\rho=1}^{\infty} \Re\left\{\bar{B}_{T, S \rho}+\bar{B}_{T, P M \rho}\right\} .
$$

with the stator contribution:

$$
\begin{aligned}
\bar{B}_{T, S \rho} & =\left[\frac{2 \mu_{0} L R_{g}}{w_{\text {tooth }}} \frac{\sin \left(\rho \frac{\Delta_{\text {slot }}}{2}\right)}{\rho} \frac{3 N q}{\pi \delta} \frac{K_{a \rho}}{\rho}\right] \bar{i}_{\rho}^{S} e^{-j \rho \vartheta_{T}}= \\
& =K_{S \rho} \bar{i}_{\rho}^{S} e^{-j \rho \vartheta_{T}},
\end{aligned}
$$

and the rotor contribution:

$$
\begin{aligned}
\bar{B}_{T, P M \rho}= & {\left[\frac{2 \mu_{0} L R_{g}}{w_{\text {tooth }}} \frac{\sin \left(\rho \frac{\Delta_{\text {slot }}}{2}\right)}{\rho} \frac{4 B_{r} \delta_{P M}}{\mu_{P M} \pi \delta} \frac{\sin \left(\rho \Delta_{P M} / 2\right)}{\rho}\right] } \\
& e^{j \rho \vartheta_{r}} e^{-j \rho \vartheta_{T}}=K_{P M \rho} e^{j \rho\left(\vartheta_{r}-\vartheta_{T}\right)}
\end{aligned}
$$

with $K_{S \rho}$ and $K_{P M \rho}$ constants depending from the harmonic order.
In case of an MTPA current control algorithm the stator contributions can be expressed considering for (4) as:

$$
\begin{array}{ll}
\bar{B}_{T, S \rho}=j K_{S \rho} i_{q} e^{j\left(\vartheta_{r}-\rho \vartheta_{T}\right)} & \text { if } \rho=3 n+1, \\
\bar{B}_{T, S \rho}=-j K_{S \rho} i_{q} e^{-j\left(\vartheta_{r}+\rho \vartheta_{T}\right)} & \text { if } \rho=3 n-1, \\
\bar{B}_{T, S \rho}=0 & \text { if } \rho=3 n .
\end{array}
$$

with $n=0,2,4, \ldots, \infty$.

At steady state operation, with an angular speed of the rotor $\omega\left(\vartheta_{r}=\omega t\right)$, the flux density in each $T^{t h}$ tooth varies in the time according to the following equation:

$$
\begin{aligned}
B_{T} & =\sum_{\rho=1}^{\infty} K_{P M \rho} \cos \left(\rho\left(\omega t-\vartheta_{T}\right)\right)- \\
& -\sum_{n=0}^{\infty} K_{S,[3 n+1]} i_{q} \sin \left(\omega t-[3 n+1] \vartheta_{T}\right)- \\
& -\sum_{n=2}^{\infty} K_{S,[3 n-1]} i_{q} \sin \left(\omega t+[3 n-1] \vartheta_{T}\right) .
\end{aligned}
$$

From the first term of (14) it is possible to note that the magnets generate in each tooth infinite time harmonics (at angular frequency $\rho \omega)$. These present the same amplitude in each tooth and they are shifted with a fixed time delay $d t=\frac{\vartheta_{T}}{\omega}$. Therefore, the behaviour of the teeth is completely symmetrical in time.

Instead, the flux density contribution produced by the currents to each tooth is composed by a sum of sinusoidal terms all at the same angular frequency $(\omega)$. In this case every component presents a time delay, from one tooth to the consecutive one, which depends on the field harmonic order (space harmonic): $d t= \pm \frac{\rho \vartheta_{T}}{\omega}$. Therefore, the resulting flux density component is sinusoidal at angular frequency $(\omega)$, but it presents a different magnitude and phase depending on the considered tooth. It is worth to note that under the assumption of sinusoidally distributed winding (i.e., sinusoidal flux distribution of the armature field in the air-gap), the latter phenomenon does not appear, and (14) can be simplified as:

$$
B_{T}=\sum_{\rho=1}^{\infty} K_{P M \rho} \cos \left(\rho\left(\omega t-\vartheta_{T}\right)\right)-K_{S, 1} \sin \left(\omega t-\vartheta_{T}\right)
$$

This highlights that the asymmetrical distribution of flux density in the stator teeth is due to the higher order harmonics of the armature field. In Fig. 2 the expected waveforms of the flux density produced by the magnets and the stator currents are shown, respectively, for three consecutive teeth when the machine has $q>1$ (in this case $q=3$ ). The corresponding Fourier spectrum is given in Fig. 3 in terms of time harmonics with respect to the fundamental, with the current and magnet contributions. Whereas the contribution of each permanent magnet spatial field harmonic generates a different time harmonic, the ones produced by the stator result in flux density components at the fundamental frequency. The analytical model, allows to decouple the effect of each stator harmonic contribution generated at the air-gap, by (11) and (13), as shown in Fig. 4.

The same analyses are carried out for a machine with one slot per pole and per phase $(q=1)$ : in Fig. 5, flux density waveforms by currents and magnets are shown respectively, Fig. 6 displays the corresponding Fourier spectra and Fig. 7 shows the stator spatial harmonic contributions. 


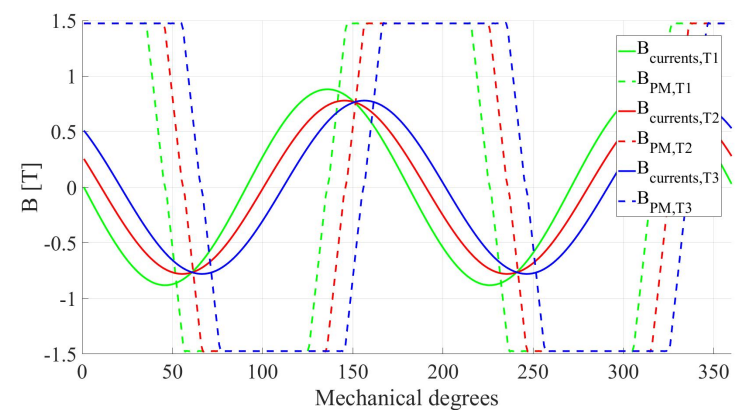

Fig. 2. Analytical flux density evaluation in the centre of three consecutive stator teeth, as a function of the rotor mechanical position $\vartheta_{r} / p=0: 360$, for 3 slot per pole and per phase $q=3$. The stator and rotor contributions are considered separately.
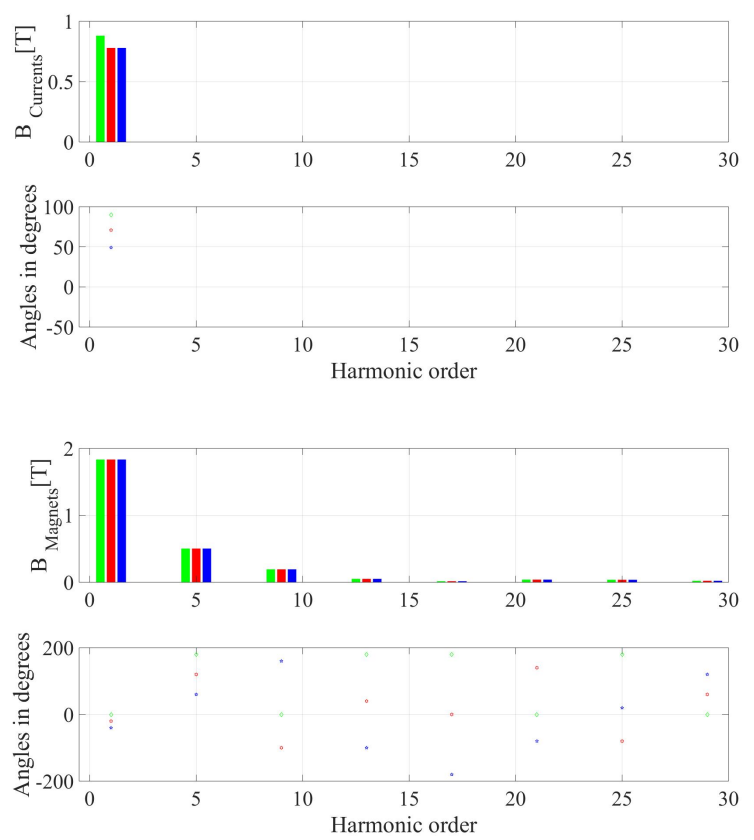

Fig. 3. Analytical evaluation of the time FFT for the stator (top) and rotor (bottom) contributions to the flux density in three consecutive teeth for a three slot per pole and per phase machine $q=3$ ).
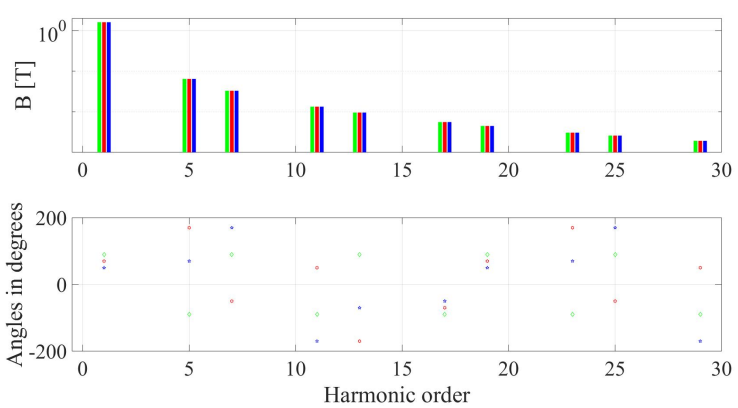

Fig. 4. Analytical evaluation of the contributions, due to different armature space harmonics generated at the air-gap, to the stator flux density in three consecutive teeth for a machine with 3 slots per pole and per phase $q=3$. The sum of all the contributions (with same frequency and different phase angles) generates the result provided in Fig. 3 (top).

As expected from the analytical model, the only case with the harmonic contributions equally shifted among the teeth is for $q=1$

The angular phase shift of the flux density in one tooth

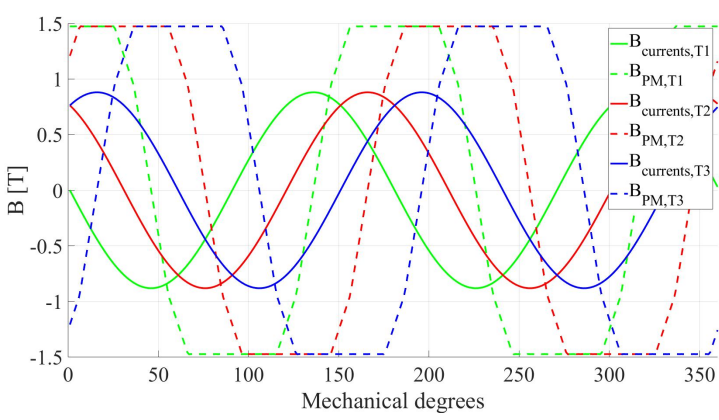

Fig. 5. Analytical flux density evaluation in the centre of three consecutive stator teeth, as a function of the rotor mechanical position $\vartheta_{r} / p=0: 360$, for 1 slot per pole and per phase $q=1$. The stator and rotor contributions are considered separately.
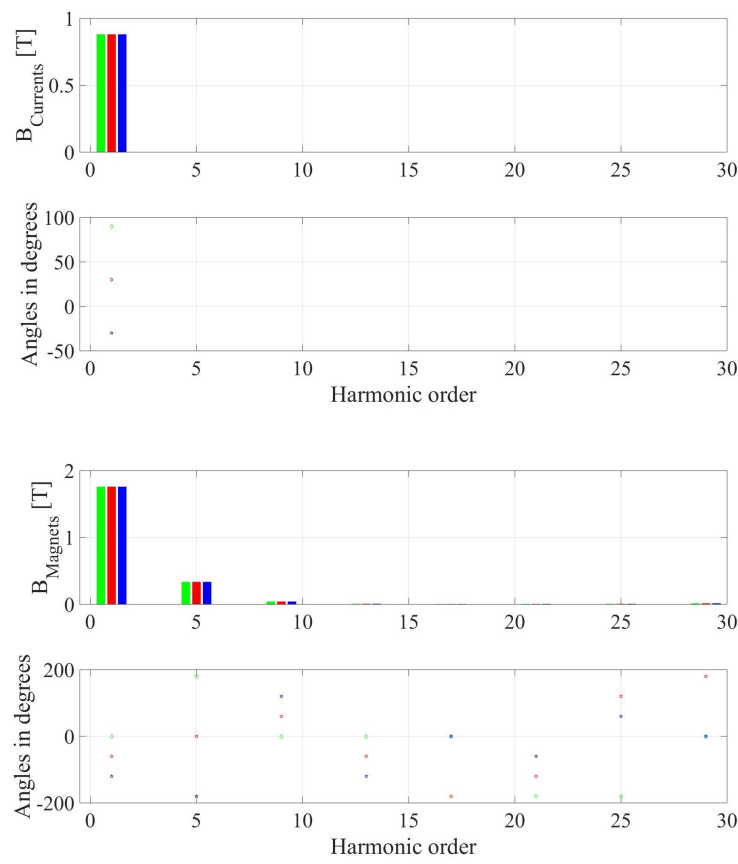

Fig. 6. Analytical evaluation of the time FFT for the stator (top) and rotor (bottom) contributions to the flux density in three consecutive teeth for a three slot per pole and per phase machine $q=1$ ).
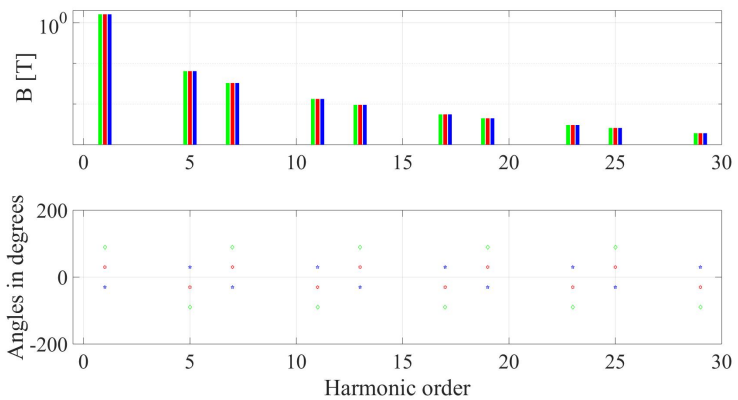

Fig. 7. Analytical evaluation of the contributions, due to different armature space harmonics generated at the air-gap, to the stator flux density in three consecutive teeth for a machine with 1 slot per pole and per phase $q=1$. The sum of all the contributions (with same frequency and equal, or opposite, phase angles) generates the result provided in Fig. 6 (top).

caused by the $\rho^{t h}$ space harmonic $\left(\rho \vartheta_{T}\right)$ can be rewritten considering that $\rho$ is equal to $3 n \pm 1$, with $n$ an even number ( $n=2 k$ ) and $N_{\text {slot }}=6 p q$. Therefore, it is possible to re-write 


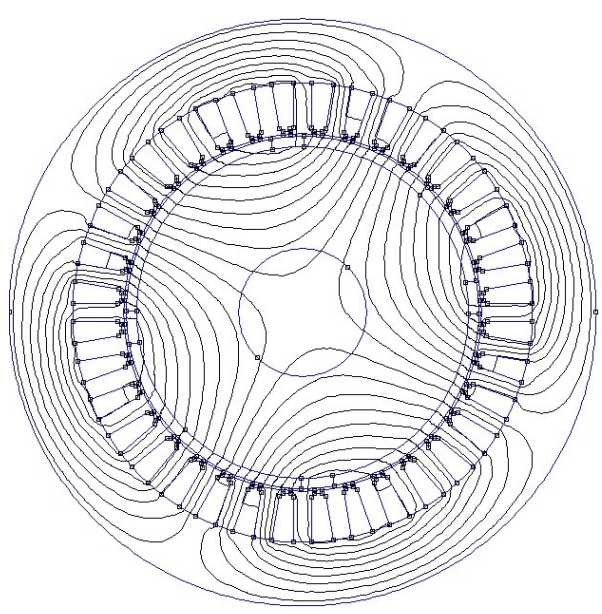

Fig. 8. SPM machine geometry with $\mathrm{q}=3$.
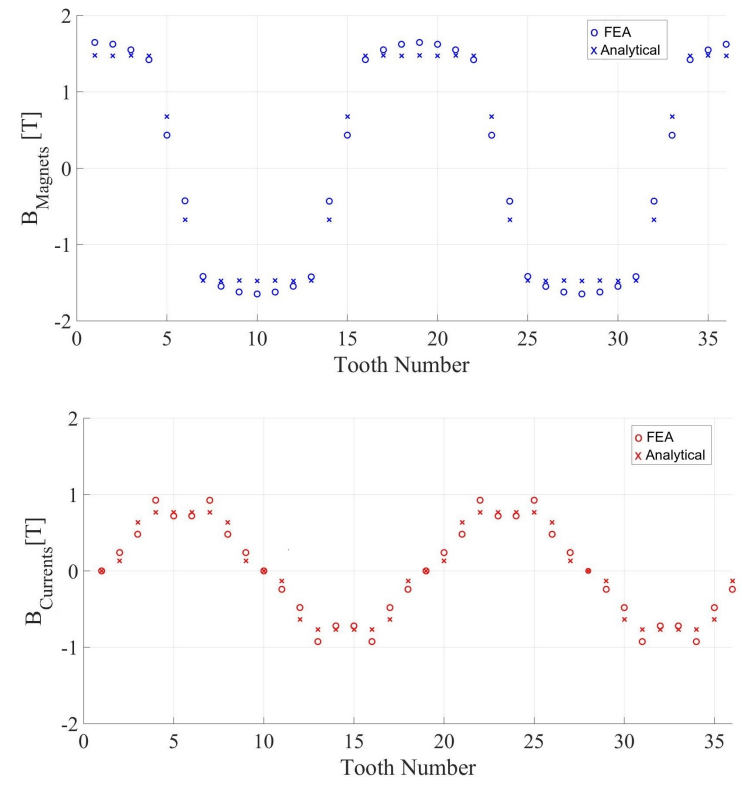

Fig. 9. Flux density for each tooth when the rotor is aligned with the phase A $\left(\vartheta_{r}=0\right)$ and the stator currents are controlled to generate the rated torque for the machine with $\mathrm{q}=3$.

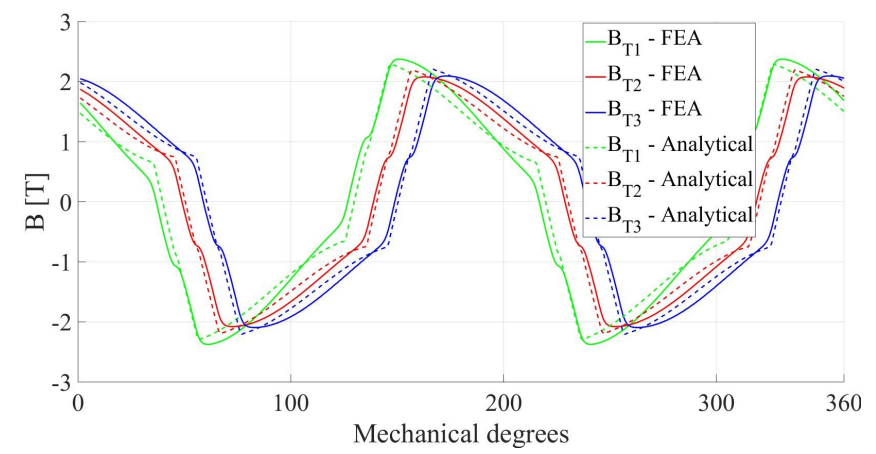

Fig. 10. Flux density comparison for three consecutive teeth between FEA and analytical approach with $\mathrm{q}=3$.

the angular phase shift as follows:

$$
\begin{aligned}
& \rho \vartheta_{T}=\rho \frac{2 \pi p}{N_{\text {slots }}}(T-1)=(3 n \pm 1) \frac{2 \pi p}{6 p q}(T-1)= \\
& =(6 k \pm 1) \frac{2 \pi}{6 q}(T-1)= \\
& =\frac{2 \pi k}{q}(T-1) \pm \frac{2 \pi}{6 q}(T-1), \quad k=0,1,2, \ldots, \infty .
\end{aligned}
$$

It can be seen that the first term in (16) is a multiple of $2 \pi$ only when $q=1$, while the second term does not depend from the considered space harmonic. It results that $q=1$ is the only solution for which the phase shift is not affected by the harmonic order.

\section{FEA VALIDATION AND RESUlts}

In order to validate the analytical model, the obtained results have been compared with the flux density values determined by means of FE simulations. The comparison is carried out considering only the radial component of the flux density at the centre of each tooth. This is a good approximation for the analysis of asymmetries among different teeth, neglecting local effects. To compare the FE results with the analytical model, linear materials have been considered [11]. As a case study, two SPM machines, with $q=3$ and $q=1$ (the parameters are listed in Table I), have been considered. The two machine geometries are shown in 8 and 11 respectively. Firstly, the 36 slots machine (with $q=3$ ) is analysed. Fig. 9 shows the comparison between FE and analytical results for the flux density distribution in all the 36 stator teeth. The magnet and current contributions (presented at the top and bottom, respectively), when the rotor is at its initial position $\left(\vartheta_{r}=0\right)$, are considered separately. Fig. 12 displays the same results obtained for the machine with 12 teeth $(q=1)$. To compare the behaviour for all the rotor positions, Fig. 10 and Fig. 13 show the distribution of flux density in three consecutive teeth for one mechanical revolution of the rotor $\left(\vartheta_{r}=0: 360\right)$, for $q=3$ and $q=1$, respectively. The results presented are validating the qualitative behaviour expected by the analytical model, i.e., the presence of asymmetries in the flux density distribution among the stator teeth when $q>1$.

\section{CONCLUSION}

In this work, a detail investigation of the flux density distribution in the teeth of an SPM machine has been carried out via analytical and FE analyses. The results highlight that in a three-phase machine with distributed winding the flux density distribution is not symmetrical when the number of slots per pole and per phase is higher than one $(q>1)$. This aspect can be taken into account in the early stage of the machine design, when simplified analytical models are used to predict the initial geometrical parameters of the electrical machine. In particular, the presented model can be used to predict the effects of these parameters on the asymmetrical distribution of iron losses and saturations, which are related to the teeth flux density. The behaviour, expected by the simplified analytical model, has been validated against FE results for two examples of SPM machines with different numbers of slots per pole and per phase: $q=3$ and $q=1$. It is found that the flux density in different teeth is asymmetrical for a machine with $q=3$, while it is symmetrical for a machine with $q=1$.

\section{REFERENCES}

[1] V. Madonna, A. Walker, P. Giangrande, G. Serra, C. Gerada, and M. Galea, "Improved thermal management and analysis for stator end-windings of electrical machines," IEEE Transactions on Industrial Electronics, vol. 66, no. 7, pp. 5057-5069, July 2019.

[2] F. Chai, P. Liang, Y. Pei, and S. Cheng, "Magnet shape optimization of surface-mounted permanent-magnet motors to reduce harmonic iron losses," IEEE Transactions on Magnetics, vol. 52, no. 7, pp. 1-4, July 2016. 


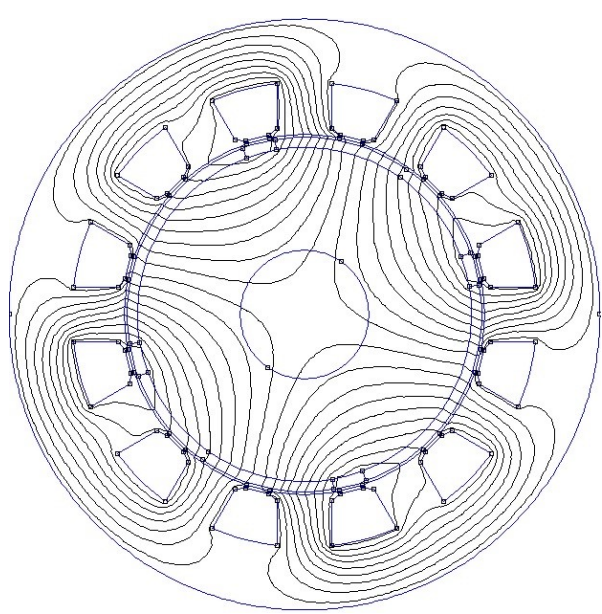

Fig. 11. SPM machine geometry with $\mathrm{q}=1$.
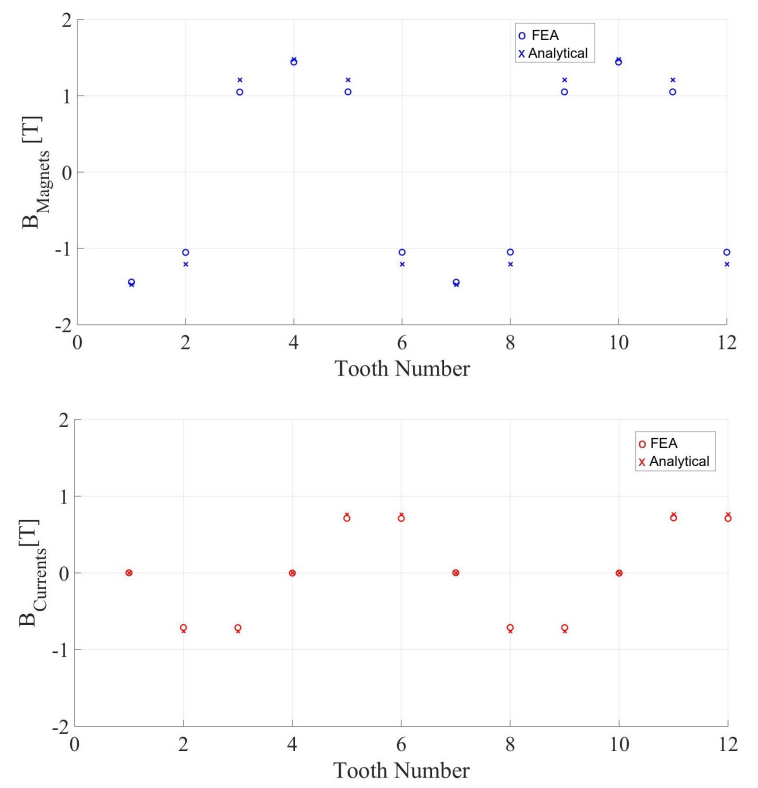

Fig. 12. Flux density for each tooth when the rotor is aligned with the phase A $\left(\vartheta_{r}=0\right)$ and the stator currents are controlled to generate the rated torque for the machine with $\mathrm{q}=1$.

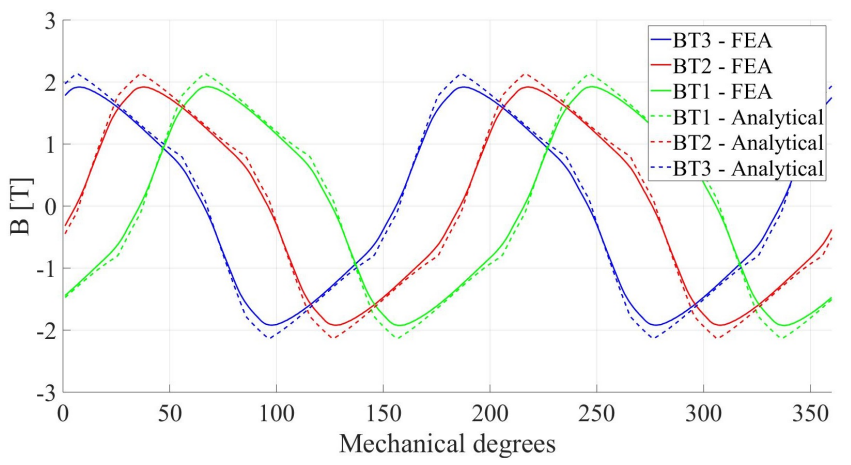

Fig. 13. Flux density comparison for three consecutive teeth between FEA and analytical approach with $\mathrm{q}=1$.
[3] S. N. Shafiqin, N. H. Halim, I. Daut, Y. Yanawati, M. Asri, M. Dina, N. Gomesh, I. Pungut, M. Abdullah, and M. N. Syatirah, "Predicting the localized flux distribution in three phase induction motor between different stator slot size," in 2011 5th International Power Engineering and Optimization Conference, June 2011, pp. 197-201.

[4] A. Al-Timimy, G. Vakil, M. Degano, P. Giangrande, C. Gerada, and M. Galea, "Considerations on the effects that core material machining has on an electrical machine's performance," IEEE Transactions on Energy Conversion, vol. 33, no. 3, pp. 1154-1163, Sep. 2018.

[5] Z. Q. Zhu, D. Howe, and C. C. Chan, "Improved analytical model for predicting the magnetic field distribution in brushless permanent-magnet machines," IEEE Transactions on Magnetics, vol. 38, no. 1, pp. 229-238, Jan 2002.

[6] L. J. Wu, Z. Q. Zhu, D. Staton, M. Popescu, and D. Hawkins, "An improved subdomain model for predicting magnetic field of surfacemounted permanent magnet machines accounting for tooth-tips," IEEE Transactions on Magnetics, vol. 47, no. 6, pp. 1693-1704, June 2011.

[7] L. Branz, M. Bortolozzi, and A. Tessarolo, "Analytical calculation of the no-load flux density in the stator core of slotless spm machines," in 2013 International Conference-Workshop Compatibility And Power Electronics, June 2013, pp. 244-249.

[8] V. Madonna, P. Giangrande, C. Gerada, and M. Galea, "Thermal analysis of fault-tolerant electrical machines for aerospace actuators," in pres on IET Electric Power Applications, 2018.

[9] P. Giangrande, V. Madonna, G. Sala, A. Kladas, C. Gerada, and M. Galea, "Design and testing of pmsm for aerospace ema applications," in IECON 2018 - 44th Annual Conference of the IEEE Industrial Electronics Society, Oct 2018, pp. 2038-2043.

[10] S. A. Odhano, P. Giangrande, R. I. Bojoi, and C. Gerada, "Selfcommissioning of interior permanent- magnet synchronous motor drives with high-frequency current injection," IEEE Transactions on Industry Applications, vol. 50, no. 5, pp. 3295-3303, Sep. 2014.

[11] A. Al-Timimy, P. Giangrande, M. Degano, M. Galea, and C. Gerada, "Comparative study of permanent magnet-synchronous and permanent magnet-flux switching machines for high torque to inertia applications," in 2017 IEEE Workshop on Electrical Machines Design, Control and Diagnosis (WEMDCD), April 2017, pp. 45-51.

\section{BIOGRAPHIES}

Giacomo Sala was born in Vercelli, Italy, in 1990. He received the B. Sc. in Power Engineering in 2012 the M. Sc. degree with honors in Electrical Engineering in 2014, and the $\mathrm{Ph}$. D. in Electrical Machines and Drives in 2018 from the University of Bologna, Bologna, Italy. He is currently a researcher of the Department of Electrical and Electronic Engineering, Faculty of Engineering, The University of Nottingham, Nottingham. His research interests include design, modelling and control of multiphase machines for high power systems with high performance and reliability, fault tolerant controls and fault diagnosis of electrical drives.

Daniele De Gaetano received the Laurea degree in Electrical Engineering from the University of Napoli "Federico II", Napoli, Italy, in 2016 and the Laurea Magistrale degree in Electrical Energy Engineering from the University of Padova, Padova, Italy, in 2018. Currently, he is a PhD Student at University of Nottingham in the Power Electronics, Machines and Control (PEMC) Group. His main research topic is on design, analysis and applications of three-phase and multi three-phase electrical machines.

Michele Degano (M'15) received the Laurea degree in electrical engineering from the University of Trieste, Trieste, Italy, in 2011, and the Ph.D. degree in industrial engineering from the University of Padova, Padova, Italy, in 2015. In 2015, he joined the Power Electronics, Machines and Control (PEMC) Group, The University of Nottingham, Nottingham, U.K., as a Research Fellow, where he is currently an Assistant Professor teaching advanced courses on electrical machines. His main research interests include design and optimization of permanent-magnet machines, reluctance and permanent-magnet-assisted synchronous reluctance motors through genetic optimization techniques, for automotive and aerospace applications.

Chris Gerada (M'05) received the Ph.D. degree in numerical modeling of electrical machines from The University of Nottingham, Nottingham, U.K., in 2005. He subsequently worked as a Researcher with The University of Nottingham on highperformance electrical drives and on the design and modeling of electromagnetic actuators for aerospace applications. Since 2006, he has been the Project Manager of the GE Aviation Strategic Partnership. In 2008, he was appointed as a Lecturer in electrical machines; in 2011, as an Associate Professor; and in 2013, as a Professor at The University of Nottingham. His main research interests include the design and modeling of high-performance electric drives and machines. Prof. Gerada serves as an Associate Editor for the IEEE TRANSACTIONS ON INDUSTRY APPLICATIONS and is the past Chair of the IEEE IES Electrical Machines Committee. 